\title{
Synthesis and Anticancer Evaluation of 1,3,4-Oxadiazoles, 1,3,4-Thiadiazoles, 1,2,4-Triazoles and Mannich Bases
}

\author{
Nadia Youssef Megally Abdo*,a and Mona Monir Kamel ${ }^{b}$ \\ ${ }^{a}$ Chemistry Department, Faculty of Education, Alexandria University; Alexandria 21526, Egypt: and ${ }^{b}$ Pharmaceutical \\ Organic Chemistry Department, Faculty of Pharmacy, Cairo University; Cairo 11562, Egypt.
}

Received January 20, 2015; accepted March 2, 2015

A series of 5-(pyridin-4-yl)- $N$-substituted-1,3,4-oxadiazol-2-amines (3a-d), 5-(pyridin-4-yl)- $N$-substituted-1,3,4-thiadiazol-2-amines (4a-d) and 5-(pyridin-4-yl)-4-substituted-1,2,4-triazole-3-thiones (5a-d) were obtained by the cyclization of hydrazinecarbothioamide derivatives $2 a-d$ derived from isonicotinic acid hydrazide. Aminoalkylation of compounds 5a-d with formaldehyde and various secondary amines furnished the Mannich bases 6a-p. The structures of the newly synthesized compounds were confirmed on the basis of their spectral data and elemental analyses. All the compounds were screened for their in vitro anticancer activity against six human cancer cell lines and normal fibroblast cells. Sixteen of the tested compounds exhibited significant cytotoxicity against most cell lines. Among these derivatives, the Mannich bases $6 \mathrm{j}$, 6m and $6 \mathrm{p}$ were found to exhibit the most potent activity. The Mannich base $6 \mathrm{~m}$ showed more potent cytotoxic activity against gastric cancer NUGC $\left(I_{50}=0.021 \mu \mathrm{M}\right)$ than the standard CHS $828\left(\mathrm{IC}_{50}=0.025 \mu \mathrm{M}\right)$. Normal fibroblast cells WI38 were affected to a much lesser extent $\left(\mathrm{IC}_{50}>10 \mu \mathrm{M}\right)$.

Key words anticancer activity; Mannich base; 1,2,4-triazole; 1,3,4-oxadiazole; 1,3,4-thiadiazole; isonicotinic acid hydrazide

About 13\% deaths of human beings throughout the world are caused by cancer, which is characterized by uncontrolled cell growth, metastasis, and invasion. ${ }^{1)}$ Among the existing cancer therapies, chemotherapy has turned out to be one of the most significant treatments in cancer management. ${ }^{2)}$ However, the most important clinical problem related to the use of new therapeutic strategies is the high toxicity of new potentially cytotoxic compounds. Thus in the light of existing problems in cancer therapy, discovery of novel, efficient, safe and selective anticancer agents is a thrust area for medicinal chemists. ${ }^{3)}$

The ring-closure reactions of carbohydrazides to prepare 1,3,4-oxadiazoles, 1,3,4-thiadiazoles and 1,2,4-triazoles are well-known and have been thoroughly studied. 1,3,4-Oxadiazoles are an important class of heterocyclic compounds with a wide range of biological activities. ${ }^{4-6)}$ Among these, a few different substituted 1,3,4-oxadiazoles have exhibited potent

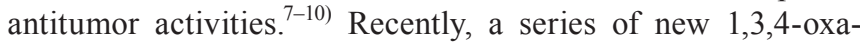
diazole derivatives incorporating a pyridine moiety were synthesized and developed as potential telomerase inhibitors. ${ }^{11)}$ On the other hand, the literature presents the variously substituted 1,3,4-thiadiazoles as compounds with a broad spectrum of biological activity. ${ }^{12-14)}$ The anticancer potential of 2-amino-1,3,4-thiadiazole derivatives has been documented by in vitro and in vivo studies. High expectations for therapies are attached to the extensive ongoing research conducted on the group of 2-amino-1,3,4-thiadiazole substituted with 2,4-dihydroxyphenyl group. ${ }^{15-17)}$

The small and simple triazole nucleus is present in compounds aimed at evaluating new entities that possess antimicrobial, antitubercular, anticonvulsant, antidepressant, antimalarial and antiinflammatory activities. ${ }^{18,19)}$ A large number of 1,2,4-triazoles have been incorporated into a wide variety of therapeutically interesting drug candidates possessing anticancer activities. ${ }^{2021)}$ Literature survey reveals that important chemotherapeutics, such as Vorozole, Letrozole and Anastrozole (Fig. 1) that consist of substituted 1,2,4-triazole ring, are currently being used for the treatment of breast cancer. ${ }^{22}$ ) Moreover, the aminoalkylation of aromatic substrates by Mannich reaction is of considerable importance for the synthesis and modification of biologically active compounds. ${ }^{23)}$ Mannich bases are common pharmacophores in the design and development of anticancer chemotherapeutic agents. ${ }^{24-27)}$ Some Mannich bases are reported to exhibit activity in vitro against Maurine P388 lymphocytic leukemia cells. ${ }^{28)}$

In the design of new compounds, development of hybrid molecules through the combination of different pharmaco-

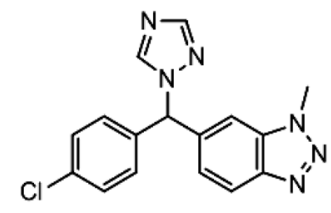

Vorozole

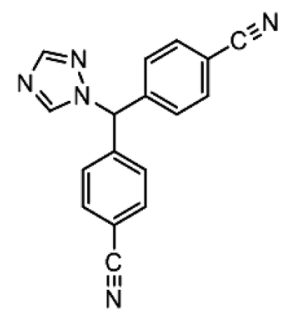

Letrozole

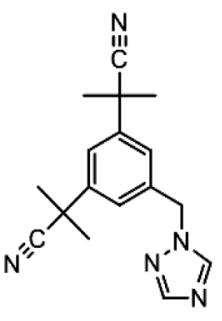

Anastrozole

Fig. 1. Chemical Structures of Breast Cancer Drugs 
<smiles>[R]Nc1nnc(-c2ccncc2)o1</smiles><smiles>[R]Nc1nnc(-c2ccncc2)s1</smiles>

Chart 1. Synthesis of Compounds $\mathbf{3 a}-\mathbf{d}$ and $\mathbf{4 a}-\mathbf{d}$

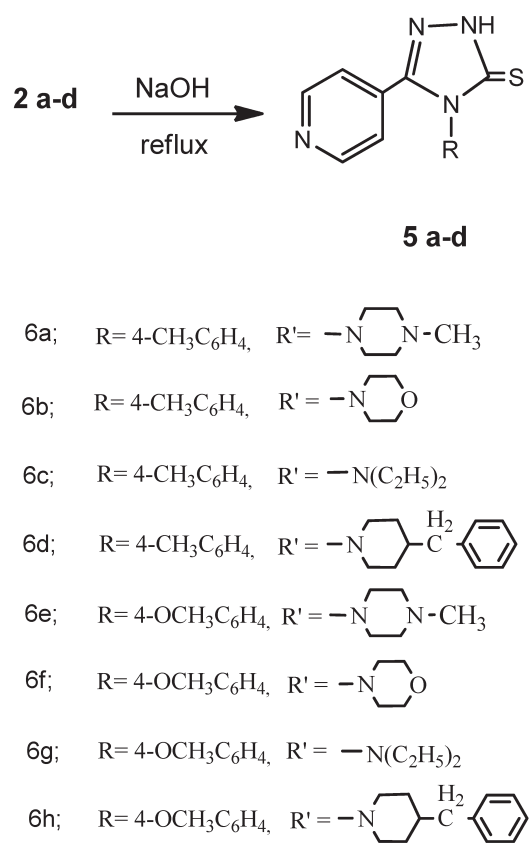

Chart 2. Synthesis of Compounds $5 \mathbf{a}-\mathbf{d}$ and $\mathbf{6 a}-\mathbf{p}$

phores in one structure may lead to compounds with increased biological activity. However, reviewing the literature reveals that the potential anticancer activity of isonicotinic acid hydrazide derivatives and Mannich bases derived from 1,2,4-triazoles have not yet been thoroughly investigated. Prompted by the aforementioned findings and in continuation of our previous work on discovering the anticancer potential of triazole derivatives ${ }^{29)}$ in the present study it was planned to synthesize hybrid compounds that comprise pyridine ring and the variant heterocyclic ring systems in order to identify new candidates that may be of value in designing new, potent, selective and less toxic anticancer agents. All the synthesized compounds were evaluated for their in vitro cytotoxicity against six human cancer cell lines and normal fibroblast cells and their structure-activity relationship (SAR) was investigated.

\section{Results and Discussion}

Chemistry The reaction sequence employed for the syn-<smiles>[R]Cn1nc(-c2ccncc2)n([R])c1=S</smiles>

6 a-p thesis of the title compounds is shown in Charts 1 and 2 . The reaction of isonicotinic acid hydrazide $\mathbf{1}$ with various aryl isothiocyanates afforded the corresponding hydrazinecarbothioamide derivatives ${ }^{30,31)} \mathbf{2 a - d}$. Oxidative cyclization of the latter compounds in $4 \mathrm{~N} \mathrm{NaOH}$ using a mixture of $\mathrm{I}_{2} /$ KI (5\%) furnished the corresponding 5-(pyridin-4-yl)- $N$-substituted-1,3,4-oxadiazol-2-amines ${ }^{32,33)}$ (3a-d) via elimination of $\mathrm{H}_{2} \mathrm{~S}$. On the other hand, 5-(pyridin-4-yl)- $N$-substituted1,3,4-thiadiazol-2-amines ${ }^{32,34)}(\mathbf{4 a}-\mathbf{d})$ were obtained by cyclization of $2 \mathbf{a}-\mathbf{d}$ upon reaction with cold conc. $\mathrm{H}_{2} \mathrm{SO}_{4}$. The structure of the synthesized compounds was confirmed on the basis of their spectral data and elemental analyses. The IR spectra of the hydrazinecarbothioamide derivatives $\mathbf{2 a - d}$ displayed the presence of signals in the range of 1672-1684 and $1249-1255 \mathrm{~cm}^{-1}$ corresponding to $\mathrm{C}=\mathrm{O}$ and $\mathrm{C}=\mathrm{S}$, respectively. Meanwhile, the ${ }^{1} \mathrm{H}-\mathrm{NMR}$ spectra of these compounds showed three singlet signals corresponding to the $\mathrm{NH}$ groups in the range of $\delta 9.71-10.82 \mathrm{ppm}$. The carbothioamides $\mathbf{2 a - d}$ 
underwent cyclization upon heating under reflux with $4 \mathrm{~N}$ aq. $\mathrm{NaOH}$ to afford 5-(pyridin-4-yl)-4-substituted-1,2,4-triazole3-thiones ${ }^{35-37)}(\mathbf{5 a}-\mathbf{d})$. The mercapto triazole derivatives are reported to be present in thione-thiol tautomeric forms in solutions. ${ }^{38)}$ The structure elucidation of the triazoles $\mathbf{5 a}-\mathbf{d}$ revealed their presence in the thione form predominantly which was further confirmed by their conversion to their Mannich bases. Thus treatment of compounds $5 \mathbf{a}-\mathbf{d}$ with each of $\mathrm{N}$ methylpiperazine, morpholine, diethyl amine and benzylpiperidine in the presence of formaldhyde solution afforded the corresponding Mannich bases 6a-p. The ${ }^{1} \mathrm{H}-\mathrm{NMR}$ spectra of the Mannich bases $\mathbf{6 a}-\mathbf{p}$ displayed additional signals due to $-\mathrm{N}-\underline{\mathrm{CH}}_{2}-\mathrm{N}-$ groups in the range of $\delta 5.25-5.78 \mathrm{ppm}$ integrating to two protons, beside signals belonging to methylpiprazine, morpholine, diethylamine or benzylpiperdine moieties. Furthermore, the ${ }^{13} \mathrm{C}$-NMR spectra of compounds $\mathbf{6 a}-\mathbf{p}$ exhibited signals in the range of $\delta 69.77-77.35 \mathrm{ppm}\left(\mathrm{N}-\underline{\mathrm{CH}}_{2}-\mathrm{N}\right)$ and $\delta$ 169.41-171.68 ppm $(\mathrm{C}=\mathrm{S})$.

In Vitro Cytotoxicity Effect on the Growth of Human Cancer Cell Lines The heterocyclic compounds, prepared in this study, were evaluated according to standard protocols for their in vitro cytotoxicity against six human cancer cell lines including cells derived from human gastric cancer (NUGC), human colon cancer (DLD1), human liver cancer (HA22T and HEPG2), nasopharyngeal carcinoma (HONE1), human breast cancer (MCF) and normal fibroblast cells (WI38). For comparison purposes, CHS 828, a pyridyl cyanoguanidine, was used as standard antitumor drug ${ }^{39)}$ (Fig. 2). The $\mathrm{IC}_{50}$ values in micro molar $(\mu \mathrm{M})$ are listed in Table 1. Sixteen of the tested compounds showed cytotoxic activity with $\mathrm{IC}_{50}$ values $<1 \mu \mathrm{M}$. The Mannich bases $\mathbf{6 j}, \mathbf{6 m}$ and $\mathbf{6 p}$ were found to be the most potent derivatives. All the synthesized compounds were tested for their cytotoxicity against normal fibroblast cells as many anticancer drugs are toxic not only against cancer cells but also normal ones. The results obtained showed that normal fibroblast cells (WI38) were affected to a much lesser extent $\left(\mathrm{IC}_{50}>10 \mu \mathrm{M}\right)$.

Structure Activity Relationship In the present study, on correlating the structures of the synthesized oxadiazoles, thiadiazoles and triazoles with their anticancer activity, it has been observed that the electronegativity of the substituents at the aromatic rings manipulated the magnitude of activity.

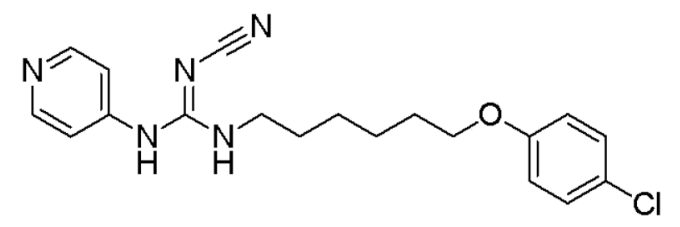

Fig. 2. Chemical Structure of CHS 828

Table 1. Cytotoxicity of Compounds $\mathbf{3 a}-\mathbf{d}, \mathbf{4 a}-\mathbf{d}, \mathbf{5 a}-\mathbf{d}$ and $\mathbf{6 a}-\mathbf{p}$ against a Variety of Cancer Cell Lines ${ }^{a)}\left[\operatorname{IC}_{50}{ }^{b)}(\mu \mathrm{M})\right]$

\begin{tabular}{|c|c|c|c|c|c|c|c|}
\hline \multirow{2}{*}{ Compound No. } & \multicolumn{7}{|c|}{ Cytotoxicity $\left(\mathrm{IC}_{50}\right.$ in $\left.\mu \mathrm{M}\right)$} \\
\hline & NUGC & DLDI & HA22T & HEPG2 & HONE1 & MCF & WI38 \\
\hline 3a & 2.312 & 1.262 & 3.274 & 1.439 & 1.330 & 2.296 & na \\
\hline $3 \mathbf{b}$ & 2.116 & 2.765 & 2.838 & 3.220 & 2.440 & 2.239 & na \\
\hline $3 c$ & 0.890 & 0.725 & 1.372 & 1.184 & 1.964 & 1.758 & na \\
\hline 3d & 1.220 & 1.833 & 2.257 & 1.277 & 2.129 & 1.320 & na \\
\hline $4 a$ & 1.142 & 2.222 & 1.348 & 1.328 & 1.820 & 2.242 & na \\
\hline $4 b$ & 1.165 & 1.322 & 2.350 & 2.221 & 2.152 & 1.224 & na \\
\hline $4 c$ & 0.418 & 0.135 & 0.753 & 0.970 & 0.849 & 2.072 & na \\
\hline $4 d$ & 0.028 & 0.229 & 0.180 & 0.264 & 0.887 & 2.290 & 1.330 \\
\hline $5 a$ & 2.286 & 1.245 & 2.631 & 2.128 & 2.112 & 3.140 & na \\
\hline $5 \mathbf{b}$ & 1.249 & 2.220 & 3.218 & 1.550 & 2.466 & 2.693 & 0.120 \\
\hline $5 c$ & 0.338 & 0.538 & 0.820 & 0.444 & 1.528 & 0.818 & na \\
\hline $5 d$ & 0.320 & 0.749 & 0.194 & 0.499 & 2.871 & 0.840 & na \\
\hline $6 a$ & 2.230 & 2.598 & 2.188 & 2.772 & 2.280 & 1.265 & 480 \\
\hline $6 b$ & 1.150 & 1.065 & 2.160 & 2.349 & 1.059 & 2.828 & na \\
\hline $6 c$ & 3.230 & 3.028 & 3.212 & 2.026 & 2.129 & 0.188 & 0.48 \\
\hline 6d & 1.288 & 1.092 & 1.739 & 1.488 & 2.879 & 1.702 & na \\
\hline $6 e$ & 1.135 & 2.160 & 2.160 & 0.814 & 0.780 & 1.286 & na \\
\hline $6 f$ & 2.340 & 1.792 & 2.826 & 2.646 & 1.174 & 3.932 & na \\
\hline $6 \mathrm{~g}$ & 2.218 & 1.346 & 2.620 & 3.837 & 2.341 & 3.280 & na \\
\hline $6 \mathrm{~h}$ & 0.380 & 0.410 & 0.128 & 0.089 & 1.890 & 0.082 & 2.199 \\
\hline $6 i$ & 0.482 & 0.139 & 0.280 & 0.520 & 1.179 & 1.108 & na \\
\hline $6 \mathbf{j}$ & 0.040 & 0.066 & 0.219 & 0.028 & 1.090 & 0.320 & na \\
\hline $6 k$ & 0.860 & 0.120 & 0.543 & 0.390 & 1.160 & 1.051 & 2.170 \\
\hline 61 & 1.127 & 0.269 & 0.324 & 1.093 & 0.549 & 0.125 & na \\
\hline $6 \mathrm{~m}$ & 0.021 & 0.044 & 0.320 & 0.066 & 0.880 & 0.338 & na \\
\hline 6n & 0.328 & 0.246 & 0.234 & 0.185 & 0.129 & 1.267 & na \\
\hline 60 & 1.350 & 1.160 & 2.290 & 2.120 & 1.126 & 0.048 & na \\
\hline $6 p$ & 0.028 & 0.036 & 0.128 & 0.439 & 0.018 & 1.120 & 2.219 \\
\hline CHS 828 & 0.025 & 2.315 & 2.067 & 1.245 & 0.015 & 0.018 & na \\
\hline
\end{tabular}

a) NUGC, gastric cancer; DLDI, colon cancer; HA22T and HEPG2, liver cancer; HONEI, nasopharyngeal carcinoma; MCF, breast cancer; WI38, normal fibroblast cells b) The sample concentration that produces a $50 \%$ reduction in cell growth. 
Thus, compounds bearing 4-chlorophenyl and 4-bromophenyl pharmacophores were found to be the only active compounds. Among the oxadiazole derivatives $\mathbf{3 a}-\mathbf{d}$, only compound $\mathbf{3 c}$ showed selective moderate activity against gastric and colon cancer cell lines. On the other hand, 1,3,4-thiadiazole derivatives $\mathbf{4 c}$ and $\mathbf{4 d}$ exhibited broad spectrum activity. Compound 4d demonstrated almost equipotent activity against gastric cancer cell line NUGC $\left(\mathrm{IC}_{50}=0.028 \mu \mathrm{M}\right)$ compared to the standard CHS 828, 4d also showed almost four fold higher activity against liver cancer cell lines compared to its 4-chlorophenyl substituted analog $\mathbf{4 c}$. The superior cytotoxic activity of the 1,3,4-thiadiazole derivatives compared to those of their bioisosters 1,2,4-oxadiazoles and 1,3,4-oxadiazoles was previously reported. ${ }^{3)}$ On the other hand, the 4-bromophenyl substituted mercaptotriazole $\mathbf{5} \mathbf{d}$ showed more potent activity against liver cancer HA22T than 5c.

To verify the effects of structural modification on the anticancer activity, sixteen Mannich bases $\mathbf{6 a}-\mathbf{p}$ incorporating methylpiperazine, morpholine, diethylamine and benzylpiperidine moieties were synthesized starting from the 1,2,4-triazoles $\mathbf{5 a}-\mathbf{d}$. The screening results again revealed the superior anticancer activity of the 4-chlorophenyl and 4-bromophenyl substituted derivatives compared to their $p$-tolyl and 4-methoxyphenyl congeners. The $p$-tolyl derivatives proved to be almost devoid of activity, while only one 4-methoxyphenyl derivative was active.

Methylpiperazine and morpholine derived Mannich bases were found to display significant cytotoxic activity. The 4-bromophenyl derivative $\mathbf{6 m}$ incorporating a methylpiperazine moiety, showed more potent cytotoxic activity against gastric cancer NUGC $\left(\mathrm{IC}_{50}=0.021 \mu \mathrm{M}\right)$ compared to the standard CHS 828 and exhibited significant activity against colon cancer DLDI and liver cancer HEPG2. Meanwhile, the 4-chlorophenyl derivative $\mathbf{6 j}$ incorporating a morpholine moiety, was found to be the most potent among all the tested Mannich bases against liver cancer HEPG2 $\left(\mathrm{IC}_{50}=0.028 \mu \mathrm{M}\right)$.

Considering the Mannich bases bearing a diethylamino moiety only the 4-chlorophenyl derivative $6 \mathbf{k}$ showed cytotoxic activity against four cell lines while $\mathbf{6 c}$ and $\mathbf{6 o}$ exhibited selective activity against breast cancer MCF.

Among the 4-methoxyphenyl Mannich bases, cytotoxic activity was displayed only by the benzyl piperidine derivative 6h. This dramatic increase in activity may be attributed to the presence of the benzyl moiety. Moreover, the 4-bromophenyl derivative 6p exhibited almost equipotent cytotoxic activity against gastric NUGC and nasopharyngeal carcinoma HONE1 compared to the standard CHS 828 as well as the highest potency against colon cancer.

From the previous findings, it might be concluded that the presence of the bioactive methylpiprazine, morpholine and benzyl piperidine moieties play an important role in displaying cytotoxic activity. Thus it is obvious that while some of the compounds were not the most potent, their specific activity against particular cell lines makes them of interest for further development as anticancer drugs.

\section{Conclusion}

This study presented the synthesis and characterization of a series of oxadiazoles, thiadiazoles, triazoles and sixteen Mannich bases derived from 1,2,4-triazoles. All the synthesized compounds were evaluated for their in vitro anticancer activ- ity against six human cancer cell lines and normal fibroblast cells and their SAR was investigated. The screening results revealed that the presence of the 4-chlorophenyl and 4-bromophenyl substituents was essential for activity. The Mannich bases demonstrated promising cytotoxic activity. Most of the compounds that exhibited anticancer activity were nontoxic to normal fibroblast cells suggesting that they may serve as lead compounds for further development of new drugs.

\section{Experimental}

Chemistry All melting points were determined on a Stuart apparatus and the values given are uncorrected. IR spectra $\left(\mathrm{KBr}, \mathrm{cm}^{-1}\right)$ were determined on a Shimadzu IR 435 spectrophotometer (Faculty of Pharmacy, Cairo University, Egypt). ${ }^{1} \mathrm{H}$ - and ${ }^{13} \mathrm{C}$-NMR spectra were recorded on Bruker Ascend $400 \mathrm{MHz}$ spectrophotometers (Microanalytical Unit, Faculty of Pharmacy, Cairo University, Egypt) using tetramethylsilane (TMS) as internal standard. Chemical shift values are recorded in ppm on $\delta$ scale. The electron impact (EI) mass spectra were recorded on a Hewlett Packard 5988 spectrometer (Microanalysis Center, Cairo University, Egypt). Elemental analyses were carried out at the Microanalysis Center, Cairo University, Egypt; found values were within $\pm 0.35 \%$ of the theoretical ones. Progress of the reactions was monitored using thin layer chromatography (TLC) sheets precoated with UV fluorescent silica gel Merck 60F 254 and were visualized using UV lamp.

General Method for Preparation of 2a-d A mixture of isonicotinic acid hydrazide $1(0.01 \mathrm{~mol})$ and the appropriate $\mathrm{p}$ substituted phenyl isothiocyanate $(0.01 \mathrm{~mol})$ in ethanol $(40 \mathrm{~mL})$ was heated under reflux for $1 \mathrm{~h}$. The reaction mixture was cooled and the obtained solid was filtered, washed and crystallized from ethanol.

2-Isonicotinyl- $N$-(4-methylphenyl)hydrazinecarbothioamide $^{30,31)}$ (2a) Yield: 95\%; mp: $176-178^{\circ} \mathrm{C}$; IR (KBr, $\left.\mathrm{cm}^{-1}\right)$ : 3275-3111 (3NH), $1681(\mathrm{C}=\mathrm{O}), 1255(\mathrm{C}=\mathrm{S}) ;{ }^{1} \mathrm{H}-\mathrm{NMR}$ $\left(\mathrm{DMSO}-d_{6}\right) \delta: 2.48$ (s, 3H, $\left.\mathrm{CH}_{3}\right), 7.11-7.28$ (m, 4H, Ar-H), 7.83 (d, $2 \mathrm{H}, J=5.9 \mathrm{~Hz}$, pyridine $\mathrm{H}-3, \mathrm{H}-5), 8.75$ (d, 2H, $J=5.9 \mathrm{~Hz}$, pyridine $\mathrm{H}-2, \mathrm{H}-6), 9.72,9.78$, and 10.81 (3s, 3H, NH, $\mathrm{D}_{2} \mathrm{O}$ exchangeable); MS (EI): $m / z(\%) 286\left(\mathrm{M}^{+}, 29\right)$. Anal. Calcd for $\mathrm{C}_{14} \mathrm{H}_{14} \mathrm{~N}_{4} \mathrm{OS}$ : C, 58.72; H, 4.93; N, 19.57. Found: C, 58.94; H, $4.63 ; \mathrm{N}, 19.25$.

2-Isonicotinyl- $N$-(4-methoxyphenyl)hydrazinecarbothioamide $^{30,31)}$ (2b) Yield: 92\%; mp: $170-172^{\circ} \mathrm{C}$; IR (KBr, $\left.\mathrm{cm}^{-1}\right)$ : 3259-3109 (3NH), $1674(\mathrm{C}=\mathrm{O}), \mathrm{C}=\mathrm{S}$ (1249); ${ }^{1} \mathrm{H}-\mathrm{NMR}$ $\left(\mathrm{DMSO}-d_{6}\right) \delta: 3.73\left(\mathrm{~s}, 3 \mathrm{H}, \mathrm{OCH}_{3}\right), 6.88-7.38(\mathrm{~m}, 4 \mathrm{H}, \mathrm{Ar}-\mathrm{H})$, $7.84(\mathrm{~d}, 2 \mathrm{H}, J=5.9 \mathrm{~Hz}$, pyridine $\mathrm{H}-3, \mathrm{H}-5), 8.75$ (d, 2H, $J=5.9 \mathrm{~Hz}$, pyridine $\mathrm{H}-2, \mathrm{H}-6), 9.71,9.76$ and $10.82(3 \mathrm{~s}, 3 \mathrm{H}$, $\mathrm{NH}, \mathrm{D}_{2} \mathrm{O}$ exchangeable); MS (EI): $m / z(\%) 302\left(\mathrm{M}^{+}, 39\right)$. Anal. Calcd for $\mathrm{C}_{14} \mathrm{H}_{14} \mathrm{~N}_{4} \mathrm{O}_{2} \mathrm{~S}$ : C, 55.61; H, 4.67; N, 18.53. Found: C, 55.42; H, 4.33; N, 18.79 .

2-Isonicotinyl- $N$-(4-cholorophenyl)-2-isonicotionylhydrazinecarbothioamide $^{30,31)}$ (2c) Yield: $94 \%$; mp: $172-174^{\circ} \mathrm{C}$; IR $\left(\mathrm{KBr}, \mathrm{cm}^{-1}\right)$ : 3244-3122 (3NH), $1674(\mathrm{C}=\mathrm{O}), 1255(\mathrm{C}=\mathrm{S})$; ${ }^{1} \mathrm{H}-\mathrm{NMR}\left(\mathrm{DMSO}-d_{6}\right) \delta$ : 7.17-7.47 (m, 4H, Ar-H), 7.85 (d, 2H, $J=5.9 \mathrm{~Hz}$, pyridine $\mathrm{H}-3, \mathrm{H}-5), 8.77$ (d, $2 \mathrm{H}, J=5.9 \mathrm{~Hz}$, pyridine H-2, H-6), 9.92 (brs, 2H, NH, $\mathrm{D}_{2} \mathrm{O}$ exchangeable), 10.88 (s, $1 \mathrm{H}, \mathrm{NH}, \mathrm{D}_{2} \mathrm{O}$ exchangeable); MS (EI): $m / z(\%) 306\left(\mathrm{M}^{+}, 67\right)$. Anal. Calcd for $\mathrm{C}_{13} \mathrm{H}_{11} \mathrm{ClN}_{4} \mathrm{OS}$ : C, 50.90; H, 3.61; N, 18.26. Found: C, 50.65; H, 3.52; N, 17.98 .

2-Isonicotinyl- $N$-(4-bromophenyl)-2-isonicotinoylhydra- 
zinecarbothioamide $^{30,31)}$ (2d) Yield: 93\%; mp: $175-177^{\circ} \mathrm{C}$; IR $\left(\mathrm{KBr}, \mathrm{cm}^{-1}\right)$ : 3244-3120 (3NH), $1672(\mathrm{C}=\mathrm{O}), 1255(\mathrm{C}=\mathrm{S})$; ${ }^{1} \mathrm{H}-\mathrm{NMR}\left(\mathrm{DMSO}-d_{6}\right) \delta$ : 7.46-7.54 (m, 4H, Ar-H), $7.86(\mathrm{~d}$, $2 \mathrm{H}, J=5.9 \mathrm{~Hz}$, pyridine $\mathrm{H}-3, \mathrm{H}-5), 8.78(\mathrm{~d}, 2 \mathrm{H}, J=5.9 \mathrm{~Hz}$, pyridine $\mathrm{H}-2, \mathrm{H}-6), 9.92,9.96,10.88\left(3 \mathrm{~s}, 3 \mathrm{H}, \mathrm{NH}, \mathrm{D}_{2} \mathrm{O}\right.$ exchangeable); MS (EI): $m / z(\%) 351\left(\mathrm{M}^{+}, 100\right)$. Anal. Calcd for $\mathrm{C}_{13} \mathrm{H}_{11} \mathrm{BrN}_{4} \mathrm{OS}$ : C, 44.46; H, 3.16; N, 15.95. Found: C, 44.31; H, 3.12; N, 16.15 .

General Method for Preparation of 3a-d A solution of $\mathrm{I}_{2} / \mathrm{KI}(5 \%)$ was added to the appropriate carbothioamide $\mathbf{2 a}-\mathbf{d}$ $(0.01 \mathrm{~mol})$ in $4 \mathrm{~N} \mathrm{NaOH}(20 \mathrm{~mL})$, until persistent yellow color. The reaction mixture was refluxed for $4 \mathrm{~h}$, cooled and poured onto ice cold water. The obtained solid was filtered, washed with water, dried and crystallized from ethanol.

5-(Pyridin-4-yl)- $N$ - $p$-tolyl-1,3,4-oxadiazol-2-amine ${ }^{32)}$

(3a) Yield: $83 \%$; mp: $298-300^{\circ} \mathrm{C}$; IR $\left(\mathrm{KBr}, \mathrm{cm}^{-1}\right)$ : $3481(\mathrm{NH}), 1604$ $(\mathrm{C}=\mathrm{N}) ;{ }^{1} \mathrm{H}-\mathrm{NMR}\left(\mathrm{DMSO}-d_{6}\right) \delta: 2.38\left(\mathrm{~s}, 3 \mathrm{H}, \mathrm{CH}_{3}\right), 7.24(\mathrm{~d}, 2 \mathrm{H}$, $J=5.9 \mathrm{~Hz}$, pyridine $\mathrm{H}-3, \mathrm{H}-5), 7.27-7.34$ (m, 4H, Ar-H), 8.57 (d, $2 \mathrm{H}, J=5.9 \mathrm{~Hz}$, pyridine $\mathrm{H}-2, \mathrm{H}-6), 14.34$ (s, $1 \mathrm{H}, \mathrm{NH}, \mathrm{D}_{2} \mathrm{O}$ exchangeable); MS (EI): $m / z(\%) 252\left(\mathrm{M}^{+}, 36\right)$. Anal. Calcd for $\mathrm{C}_{14} \mathrm{H}_{12} \mathrm{~N}_{4} \mathrm{O}$ : C, 66.65; H, 4.79; N, 22.21. Found: C, 66.84; H, $4.63 ; \mathrm{N}, 22.43$.

$N$-(4-Methoxyphenyl)-5-(pyridin-4-yl)-1,3,4-oxadiazol2-amine ${ }^{32)}$ (3b) Yield: $85 \%$; mp: $>300^{\circ} \mathrm{C}$; IR $\left(\mathrm{KBr}, \mathrm{cm}^{-1}\right)$ : $3471(\mathrm{NH}), 1608(\mathrm{C}=\mathrm{N}) ;{ }^{1} \mathrm{H}-\mathrm{NMR}\left(\mathrm{DMSO}-d_{6}\right) \delta: 3.81(\mathrm{~s}, 3 \mathrm{H}$, $\left.\mathrm{OCH}_{3}\right), 7.05(\mathrm{~d}, 2 \mathrm{H}, J=5.9 \mathrm{~Hz}$, pyridine $\mathrm{H}-3, \mathrm{H}-5), 7.25-7.34$ (m, 4H, Ar-H), 8.57 (d, 2H, J=5.9 Hz, pyridine H-2, H-6), 14.31 (s, 1H, NH, D $\mathrm{O}$ exchangeable); ${ }^{13} \mathrm{C}-\mathrm{NMR}$ (DMSO) $\delta$ : $55.92,115.12,122.00,122.30,127.11,130.30,133.74,149.01$, 150.55, 160.27, 169.99; MS (EI): $m / z(\%) 269$ (M+H, 16). Anal. Calcd for $\mathrm{C}_{14} \mathrm{H}_{12} \mathrm{~N}_{4} \mathrm{O}_{2}:$ C, 62.68; H, 4.51; N, 20.88. Found: C, 62.88; H, 4.64; N, 21.08.

$\mathrm{N}$-(4-Chlorophenyl)-5-(pyridin-4-yl)-1,3,4-oxadiazol-2amine $^{32)}$ (3c) Yield: 86\%; $\mathrm{mp}>300^{\circ} \mathrm{C}$; IR $\left(\mathrm{KBr}, \mathrm{cm}^{-1}\right)$ : $3446(\mathrm{NH}), 1610(\mathrm{C}=\mathrm{N})$; ${ }^{1} \mathrm{H}-\mathrm{NMR}\left(\mathrm{DMSO}-d_{6}\right) \delta: 7.26(\mathrm{~d}, 2 \mathrm{H}$, $J=5.9 \mathrm{~Hz}$, pyridine $\mathrm{H}-3, \mathrm{H}-5), 7.47-7.67$ (m, 4H, Ar-H), 8.59 (d, $2 \mathrm{H}, J=5.9 \mathrm{~Hz}$, pyridine $\mathrm{H}-2, \mathrm{H}-6), 14.39$ (s, $1 \mathrm{H}, \mathrm{NH}, \mathrm{D}_{2} \mathrm{O}$ exchangeable); MS (EI): $m / z(\%) 272\left(\mathrm{M}^{+}, 30\right)$. Anal. Calcd for $\mathrm{C}_{13} \mathrm{H}_{9} \mathrm{ClN}_{4} \mathrm{O}$ : C, 57.26; H, 3.33; N, 20.55. Found: C, 56.99; H, $3.01 ; \mathrm{N}, 20.67$.

$N$-(4-Bromophenyl)-5-(pyridin-4-yl)-1,3,4-oxadiazol2-amine ${ }^{33)}$ (3d) Yield: 81\%; mp: 223-225 ${ }^{\circ}$; IR $\left(\mathrm{KBr}, \mathrm{cm}^{-1}\right)$ : $3436(\mathrm{NH}), 1606(\mathrm{C}=\mathrm{N})$; ${ }^{1} \mathrm{H}-\mathrm{NMR}\left(\mathrm{DMSO}-d_{6}\right) \delta: 7.26(\mathrm{~d}, 2 \mathrm{H}$, $J=5.9 \mathrm{~Hz}$, pyridine H-3, H-5), 7.39-7.82 (m, 4H, Ar-H), 8.60 (d, 2H, J=5.9 Hz, pyridine $\mathrm{H}-2, \mathrm{H}-6), 14.38$ (s, $1 \mathrm{H}, \mathrm{NH}, \mathrm{D}_{2} \mathrm{O}$ exchangeable); MS (EI): $m / z(\%) 317\left(\mathrm{M}^{+}, 17\right)$. Anal. Calcd for $\mathrm{C}_{13} \mathrm{H}_{9} \mathrm{BrN}_{4} \mathrm{O}: \mathrm{C}, 49.23 ; \mathrm{H}, 2.86 ; \mathrm{N}, 17.67$. Found: C, 49.45; H, $2.76 ; \mathrm{N}, 17.54$.

General Method for Preparation of $4 \mathbf{a}-\mathbf{d}$ The appropriate carbothioamide $\mathbf{2 a}-\mathbf{d}(0.01 \mathrm{~mol})$ was added portion wise to cold conc. $\mathrm{H}_{2} \mathrm{SO}_{4}(10 \mathrm{~mL})$ with stirring. The solution was kept at room temperature for $2 \mathrm{~h}$ and then poured onto ice-cold water. The reaction mixture was stirred and allowed to stand for $1 \mathrm{~h}$. The separated orange solid was filtered, washed with water and crystallized from ethanol.

5-(Pyridin-4-yl)- $N$ - $p$-tolyl-1,3,4-thiadiazol-2-amine ${ }^{32,34)}$ (4a) Yield: $78 \%$; mp: $227-229^{\circ} \mathrm{C}$; IR $\left(\mathrm{KBr}, \mathrm{cm}^{-1}\right)$ : $3263(\mathrm{NH})$, 1633, $1608(\mathrm{C}=\mathrm{N})$; ${ }^{1} \mathrm{H}-\mathrm{NMR}\left(\mathrm{DMSO}-d_{6}\right) \delta: 2.26\left(\mathrm{~s}, 3 \mathrm{H}, \mathrm{CH}_{3}\right)$, 7.17-7.53 (m, 4H, Ar-H), 8.28 (brs, 2H, pyridine H-3, H-5), 8.89 (brs, 2H, pyridine H-2, H-6), 10.94 (s, 1H, NH, D $2 \mathrm{O}$ exchangeable); MS (EI): $m / z(\%) 268\left(\mathrm{M}^{+}, 56\right)$. Anal. Calcd for
$\mathrm{C}_{14} \mathrm{H}_{12} \mathrm{~N}_{4} \mathrm{~S}: \mathrm{C}, 62.66 ; \mathrm{H}, 4.51 ; \mathrm{N}, 20.88$. Found: C, 62.45; H, 4.32; N, 20.94.

$N$-(4-Methoxyphenyl)-5-(pyridin-4-yl)-1,3,4-thiadiazol2-amine ${ }^{32)}$ (4b) Yield: $80 \%$; mp: $>300^{\circ} \mathrm{C}$; IR $\left(\mathrm{KBr}, \mathrm{cm}^{-1}\right)$ : $3261(\mathrm{NH}), 1635,1612(\mathrm{C}=\mathrm{N}) ;{ }^{1} \mathrm{H}-\mathrm{NMR}\left(\mathrm{DMSO}-d_{6}\right) \delta: 3.73(\mathrm{~s}$, $3 \mathrm{H}, \mathrm{OCH}_{3}$ ), 6.96-7.58 (m, 4H, Ar-H), 8.02 (br s, $2 \mathrm{H}$, pyridine $\mathrm{H}-3, \mathrm{H}-5$ ), 8.77 (br s, 2H, pyridine H-2, H-6), 10.70 (s, 1H, $\mathrm{NH}, \mathrm{D}_{2} \mathrm{O}$ exchangeable); MS (EI): $m / z(\%) 284\left(\mathrm{M}^{+}, 42\right)$. Anal. Calcd for $\mathrm{C}_{14} \mathrm{H}_{12} \mathrm{~N}_{4} \mathrm{OS}$ : C, 59.14; H, 4.25; N, 19.70. Found: C, 58.99; H, 4.15; N, 19.96.

$N$-(4-Chlorophenyl)-5-(pyridin-4-yl)-1,3,4-thiadiazol2-amine ${ }^{32,34)}$ (4c) Yield: $77 \%$; mp: $238-240^{\circ} \mathrm{C}$; IR $(\mathrm{KBr}$, $\left.\mathrm{cm}^{-1}\right): 3257(\mathrm{NH}), 1633,1614(\mathrm{C}=\mathrm{N}) ;{ }^{1} \mathrm{H}-\mathrm{NMR}\left(\mathrm{DMSO}-d_{6}\right) \delta$ : 7.40-7.71 (m, 4H, Ar-H), 8.18 (br s, 2H, pyridine H-3, H-5), 8.86 (br s, 2H, pyridine H-2, H-6), 11.01 (s, 1H, NH, $\mathrm{D}_{2} \mathrm{O}$ exchangeable); MS (EI): $m / z(\%) 288\left(\mathrm{M}^{+}, 36\right)$. Anal. Calcd for $\mathrm{C}_{13} \mathrm{H}_{9} \mathrm{ClN}_{4} \mathrm{~S}$ : C, 54.07; H, 3.14; N, 19.40. Found: C, 54.26; H, $3.02 ; \mathrm{N}, 19.61$.

$N$-(4-Bromophenyl)-5-(pyridin-4-yl)-1,3,4-thiadiazol2-amine ${ }^{34)}$ (4d) Yield: 82\%; mp: $242-244^{\circ} \mathrm{C}$; IR ( $\mathrm{KBr}$, $\left.\mathrm{cm}^{-1}\right): 3267(\mathrm{NH}), 1633,1610(\mathrm{C}=\mathrm{N}) ;{ }^{1} \mathrm{H}-\mathrm{NMR}\left(\mathrm{DMSO}-d_{6}\right)$ $\delta$ : 7.52-7.63 (m, 4H, Ar-H), 7.99 (br s, 2H, pyridine H-3, H-5), 8.77 (br s, 2H, pyridine $\mathrm{H}-2, \mathrm{H}-6), 10.88$ (s, 1H, NH, $\mathrm{D}_{2} \mathrm{O}$ exchangeable); MS (EI): $m / z(\%) 333\left(\mathrm{M}^{+}, 10\right)$. Anal. Calcd for $\mathrm{C}_{13} \mathrm{H}_{9} \mathrm{BrN}_{4} \mathrm{~S}: \mathrm{C}, 46.86 ; \mathrm{H}, 2.72 ; \mathrm{N}, 16.81$. Found: C, 46.95; H, $2.56 ; \mathrm{N}, 16.64$.

General Method for Preparation of 5a-d A solution of the appropriate carbothioamide $\mathbf{2 a}-\mathbf{d}(0.01 \mathrm{~mol})$ in $1 \mathrm{~N} \mathrm{NaOH}$ $(30 \mathrm{~mL})$ was heated under reflux for $2 \mathrm{~h}$. The solution was cooled and acidified to $\mathrm{pH} 5-6$ with acetic acid. The precipitate formed was filtered, washed with water and crystallized from ethanol.

5-(Pyridin-4-yl)-4- $p$-tolyl-2H-1,2,4-triazole-3(4H)-thione ${ }^{35)}$ (5a) Yield: $85 \%$; mp: $224-226^{\circ} \mathrm{C}$; IR $\left(\mathrm{KBr}, \mathrm{cm}^{-1}\right): 2654$ $(\mathrm{SH}), 1604(\mathrm{C}=\mathrm{N}), 1284(\mathrm{C}=\mathrm{S}) ;{ }^{1} \mathrm{H}-\mathrm{NMR}\left(\mathrm{DMSO}-d_{6}\right) \delta: 2.34$ (s, 3H, $\mathrm{CH}_{3}$ ), 7.21-7.31 (m, 6H, 4 Ar-H and pyridine $\left.\mathrm{H}-3, \mathrm{H}-5\right)$, 8.52 (s, 2H, pyridine H-2, H-6); MS (EI): $m / z(\%) 268\left(\mathrm{M}^{+}\right.$, 78). Anal. Calcd for $\mathrm{C}_{14} \mathrm{H}_{12} \mathrm{~N}_{4} \mathrm{~S}: \mathrm{C}, 62.66 ; \mathrm{H}, 4.51 ; \mathrm{N}, 20.88$. Found: C, 62.79; H, 4.71; N, 20.62.

4-(4-Methoxyphenyl)-5-(pyridin-4-yl)-2H-1,2,4-triazole$3(4 H)$-thione $^{36)}(\mathbf{5 b}) \quad$ Yield: $82 \%$; mp: $218-220^{\circ} \mathrm{C}$; IR (KBr, $\left.\mathrm{cm}^{-1}\right)$ : $2561 \quad(\mathrm{SH}), \quad 1608 \quad(\mathrm{C}=\mathrm{N}), \quad 1261 \quad(\mathrm{C}=\mathrm{S}) ;{ }^{1} \mathrm{H}-\mathrm{NMR}$ $\left(\mathrm{DMSO}-d_{6}\right) \delta$ : $3.80\left(\mathrm{~s}, 3 \mathrm{H}, \mathrm{OCH}_{3}\right), 7.03-7.25(\mathrm{~m}, 4 \mathrm{H}, \mathrm{Ar}-\mathrm{H})$, 7.29 (d, 2H, $J=6 \mathrm{~Hz}$, pyridine $\mathrm{H}-3, \mathrm{H}-5), 8.56(\mathrm{~d}, 2 \mathrm{H}, J=6 \mathrm{~Hz}$, pyridine H-2, H-6); MS (EI): $m / z$ (\%) $284\left(\mathrm{M}^{+}, 100\right)$. Anal. Calcd for $\mathrm{C}_{14} \mathrm{H}_{12} \mathrm{~N}_{4} \mathrm{OS}$ : C, 59.14; H, 4.25; N, 19.70. Found: C, 58.94; H, 4.02; N, 19.89 .

4-(4-Chlorophenyl)-5-(pyridin-4-yl)-2 H-1,2,4-triazole3(4H)-thione ${ }^{37)}$ (5c) Yield: 86\%; mp: $261-263^{\circ} \mathrm{C}$; IR $(\mathrm{KBr}$, $\left.\mathrm{cm}^{-1}\right): 2551 \quad(\mathrm{SH}), \quad 1614 \quad(\mathrm{C}=\mathrm{N}), 1280 \quad(\mathrm{C}=\mathrm{S}) ;{ }^{1} \mathrm{H}-\mathrm{NMR}$ $\left(\mathrm{DMSO}-d_{6}\right) \delta: 7.26-7.49$ (m, 4H, Ar-H), 7.60 (d, 2H, J=6 Hz, pyridine $\mathrm{H}-3, \mathrm{H}-5), 8.59$ (d, 2H, $J=6 \mathrm{~Hz}$, pyridine $\mathrm{H}-2, \mathrm{H}-6)$; MS (EI): $m / z(\%) 288\left(\mathrm{M}^{+}, 100\right)$. Anal. Calcd for $\mathrm{C}_{13} \mathrm{H}_{9} \mathrm{ClN}_{4} \mathrm{~S}$ : C, 54.07; H, 3.14; N, 19.40. Found: C, 54.32; H, 3.34; N, 19.67.

4-(4-Bromophenyl)-5-(pyridin-4-yl)-2H-1,2,4-triazole3(4H)-thione ${ }^{37)}$ (5d) Yield: 84\%; mp: 298-300 ${ }^{\circ}$; IR $\left(\mathrm{KBr}, \mathrm{cm}^{-1}\right): 2559(\mathrm{SH}), 1608(\mathrm{C}=\mathrm{N}), 1290(\mathrm{C}=\mathrm{S}) ;{ }^{1} \mathrm{H}-\mathrm{NMR}$ $\left(\mathrm{DMSO}-d_{6}\right) \delta: 7.26-7.42(\mathrm{~m}, 4 \mathrm{H}, \mathrm{Ar}-\mathrm{H}), 7.73(\mathrm{~d}, 2 \mathrm{H}, J=6 \mathrm{~Hz}$, pyridine $\mathrm{H}-3, \mathrm{H}-5), 8.60$ (d, 2H, $J=6.0 \mathrm{~Hz}$, pyridine $\mathrm{H}-2, \mathrm{H}-6)$; MS (EI): $m / z(\%) 333\left(\mathrm{M}^{+}, 28\right)$. Anal. Calcd for $\mathrm{C}_{13} \mathrm{H}_{9} \mathrm{BrN}_{4} \mathrm{~S}$ : C, 46.86; H, 2.72; N, 16.81. Found: C, 46.66; H, 2.55; N; 16.64. 
General Method for Preparation of 6a-p A mixture of each of triazole $\mathbf{5 a}-\mathbf{d}(0.01 \mathrm{~mol})$, formaldehyde $(40 \%, 1.5 \mathrm{~mL})$ and the appropriate substituted secondary amine $(0.01 \mathrm{~mol})$ in dioxane $(20 \mathrm{~mL})$ was stirred for $1 \mathrm{~h}$ and left overnight at room temperature. The obtained solid was filtered, dried and crystallized from ethanol.

2-((4-Methylpiperazin-1-yl)methyl)-5-(pyridin-4-yl)-4- $p$ tolyl-2H-1,2,4-triazole-3(4H)-thione (6a) Yield: 78\%; mp: $179-181^{\circ} \mathrm{C}$; IR $\left(\mathrm{KBr}, \mathrm{cm}^{-1}\right): 1604(\mathrm{C}=\mathrm{N}), 1274 \quad(\mathrm{C}=\mathrm{S})$; ${ }^{1} \mathrm{H}-\mathrm{NMR}\left(\mathrm{DMSO}-d_{6}\right) \delta: 2.31\left(\mathrm{~s}, 3 \mathrm{H}, \mathrm{CH}_{3}\right), 2.42\left(\mathrm{~s}, 3 \mathrm{H}, \mathrm{CH}_{3}\right)$, 2.47 (s, $4 \mathrm{H}, 2 \mathrm{CH}_{2}$ of piperazine), $3.01\left(\mathrm{~s}, 4 \mathrm{H}, 2 \mathrm{CH}_{2}\right.$ of piperazine), 5.77 (s, 2H, $\mathrm{CH}_{2}$ ), 7.19-7.35 (m, 4H, Ar-H), 7.37 (d, 2H, $J=6 \mathrm{~Hz}$, pyridine $\mathrm{H}-3, \mathrm{H}-5), 8.60$ (d, $2 \mathrm{H}, J=6 \mathrm{~Hz}$, pyridine $\mathrm{H}-2, \mathrm{H}-6$ ); ${ }^{13} \mathrm{C}-\mathrm{NMR}$ (DMSO- $d_{6}$ ) $\delta$ : 21.42, 46.80, 54.72, 57.62, $77.03,121.63,127.86,130.73,130.83,131.4,132.80,140.88$, 147.29, 150.35, 169.72; MS (EI): $m / z(\%) 380\left(\mathrm{M}^{+}\right.$, 40). Anal. Calcd for $\mathrm{C}_{20} \mathrm{H}_{24} \mathrm{~N}_{6} \mathrm{~S}$ : C, 63.13; H, 6.36; N, 22.09. Found: C, 63.37; H, 6.32; N, 21.83.

2-(Morpholinomethyl)-5-(pyridin-4-yl)-4- $p$-tolyl-2H-1,2,4triazole-3(4H)-thione (6b) Yield: $75 \%$; mp: $178-180^{\circ} \mathrm{C}$; IR $\left(\mathrm{KBr}, \mathrm{cm}^{-1}\right): 1597(\mathrm{C}=\mathrm{N}), 1247(\mathrm{C}=\mathrm{S}) ;{ }^{1} \mathrm{H}-\mathrm{NMR}\left(\mathrm{DMSO}-d_{6}\right)$ $\delta: 2.44\left(\mathrm{~s}, 3 \mathrm{H}, \mathrm{CH}_{3}\right), 2.92\left(\mathrm{~s}, 4 \mathrm{H}, 2 \mathrm{CH}_{2}\right.$ morpholine), 3.66 (s, $4 \mathrm{H}, 2 \mathrm{CH}_{2}$ morpholine), $5.27\left(\mathrm{~s}, 2 \mathrm{H}, \mathrm{CH}_{2}\right), 7.17-7.32(\mathrm{~m}, 4 \mathrm{H}$, Ar-H), 7.35 (d, 2H, J=6 Hz, pyridine H-3, H-5), 8.56 (d, 2H, $J=6 \mathrm{~Hz}$, pyridine $\mathrm{H}-2, \mathrm{H}-6) ;{ }^{13} \mathrm{C}-\mathrm{NMR}\left(\mathrm{DMSO}-d_{6}\right) \delta: 21.41$, $50.81,66.88,70.12,121.59,127.83,130.75,132.00,132.29$, 140.64, 146.92, 150.34, 171.47; MS (EI): $m / z(\%) 367\left(\mathrm{M}^{+}\right.$, 54). Anal. Calcd for $\mathrm{C}_{19} \mathrm{H}_{21} \mathrm{~N}_{5} \mathrm{OS}$ : C, 62.10; H, 5.76; N, 19.06 . Found: C, 62.32; H, 5.46; N, 19.12 .

2-((Diethylamino)methyl)-5-(pyridin-4-yl)-4- $p$-tolyl-2H-1,2,4triazole-3(4H)-thione (6c) Yield: $70 \%$; mp: $168-170^{\circ} \mathrm{C}$; IR $\left(\mathrm{KBr}, \mathrm{cm}^{-1}\right) ; 1602(\mathrm{C}=\mathrm{N}), 1278(\mathrm{C}=\mathrm{S}) ;{ }^{1} \mathrm{H}-\mathrm{NMR}\left(\mathrm{DMSO}-d_{6}\right)$ $\delta: 1.23\left(\mathrm{t}, 6 \mathrm{H}, J=7.2 \mathrm{~Hz}, 2 \mathrm{CH}_{2}-\mathrm{CH}_{3}\right), 2.45\left(\mathrm{~s}, 3 \mathrm{H}, \mathrm{CH}_{3}\right), 2.92$ (q, $\left.4 \mathrm{H}, J=7.2 \mathrm{~Hz}, 2 \mathrm{CH}_{2}-\mathrm{CH}_{3}\right), 5.78\left(\mathrm{~s}, 2 \mathrm{H}, \mathrm{CH}_{2}\right), 7.19-7.28$ $(\mathrm{m}, 4 \mathrm{H}, \mathrm{Ar}-\mathrm{H}), 7.35(\mathrm{~d}, 2 \mathrm{H}, J=6 \mathrm{~Hz}$, pyridine $\mathrm{H}-3, \mathrm{H}-5), 8.56$ (d, $2 \mathrm{H}, J=6 \mathrm{~Hz}$, pyridine $\mathrm{H}-2, \mathrm{H}-6$ ); ${ }^{13} \mathrm{C}-\mathrm{NMR}$ (DMSO- $d_{6}$ ): $\delta: 13.03,21.24,45.66,71.91,121.51,127.76,130.52,131.65$, 132.81, 140.40, 147.05, 150.10, 170.60; MS (EI): $\mathrm{m} / \mathrm{z}(\%) 353$ $\left(\mathrm{M}^{+}\right.$, 55). Anal. Calcd for $\mathrm{C}_{19} \mathrm{H}_{23} \mathrm{~N}_{5} \mathrm{~S}: \mathrm{C}, 64.56 ; \mathrm{H}, 6.56 ; \mathrm{N}$, 19.81. Found: C, 64.78; H, 6.33; N, 19.72.

2-((4-Benzylpiperidin-1-yl)methyl)-5-(pyridin-4-yl)-4- $p$ tolyl-2H-1,2,4-triazole-3(4H)-thione (6d) Yield: 78\%; mp: $171-173^{\circ} \mathrm{C}$; IR $\left(\mathrm{KBr}, \mathrm{cm}^{-1}\right): 1600(\mathrm{C}=\mathrm{N}), 1267 \quad(\mathrm{C}=\mathrm{S})$; ${ }^{1} \mathrm{H}-\mathrm{NMR}\left(\mathrm{DMSO}-d_{6}\right) \delta$ : $1.29-1.71\left(\mathrm{~m}, 5 \mathrm{H}, 2 \mathrm{CH}_{2}+\mathrm{CH}\right.$ piperidine), $2.46\left(\mathrm{~s}, 3 \mathrm{H}, \mathrm{CH}_{3}\right), 2.47$ ( $\mathrm{s}, 4 \mathrm{H}, 2 \mathrm{CH}_{2}$ piperidine), $3.24\left(\mathrm{~s}, 2 \mathrm{H}, \mathrm{CH}_{2}-\mathrm{C}_{6} \mathrm{H}_{5}\right), 5.27\left(\mathrm{~s}, 2 \mathrm{H}, \mathrm{CH}_{2}\right), 7.13-7.29(\mathrm{~m}$, 9H, Ar-H), 7.34 (d, 2H, J=6 Hz, pyridine H-3, H-5), 8.57 (d, $2 \mathrm{H}, J=6 \mathrm{~Hz}$, pyridine $\mathrm{H}-2, \mathrm{H}-6) ;{ }^{13} \mathrm{C}-\mathrm{NMR}$ (DMSO- $\left.d_{6}\right) \delta$ : 21.44, 32.17, 37.44, 43.16, 51.32, 70.70, 121.56, 125.83, 127.90, $128.16,129.09$, 130.72, 132.13, 133.04, 140.53, 140.56, 146.67, 150.31, 171.28; MS (EI): $m / z(\%) 455\left(\mathrm{M}^{+}, 29\right)$. Anal. Calcd for $\mathrm{C}_{27} \mathrm{H}_{29} \mathrm{~N}_{5} \mathrm{~S}$ : C, 71.18; H, 6.42; N, 15.37 Found: C, 71.27; H, 6.32; N, 15.43 .

4-(4-Methoxyphenyl)-2-((4-methylpiperazin-1-yl)methyl)-5(pyridin-4-yl)-2H-1,2,4-triazole-3(4H)-thione (6e) Yield: 78\%; mp: $188-190^{\circ} \mathrm{C}$; IR $\left(\mathrm{KBr}, \mathrm{cm}^{-1}\right): 1600(\mathrm{C}=\mathrm{N}), 1266(\mathrm{C}=\mathrm{S})$; ${ }^{1} \mathrm{H}-\mathrm{NMR}\left(\mathrm{DMSO}-d_{6}\right) \delta: 2.31\left(\mathrm{~s}, 3 \mathrm{H}, \mathrm{CH}_{3}\right), 2.49\left(\mathrm{~s}, 4 \mathrm{H}, 2 \mathrm{CH}_{2}\right.$ of piperazine), $2.99\left(\mathrm{~s}, 4 \mathrm{H}, 2 \mathrm{CH}_{2}\right.$ of piperazine), $3.89(\mathrm{~s}, 3 \mathrm{H}$, $\mathrm{OCH}_{3}$ ), 5.32 (s, 2H, CH $), 7.03-7.21$ (m, 4H, Ar-H), 7.23 (d, $2 \mathrm{H}, J=6 \mathrm{~Hz}$, pyridine $\mathrm{H}-3, \mathrm{H}-5), 8.58(\mathrm{~d}, 2 \mathrm{H}, J=6 \mathrm{~Hz}$, pyridine $\mathrm{H}-2, \mathrm{H}-6) ;{ }^{13} \mathrm{C}-\mathrm{NMR}$ (DMSO- $d_{6}$ ) $\delta$ : 46.02, 50.25, 54.93, 55.56,
69.77, 115.24, 121.56, 127.10, 129.26, 132.94, 146.85, 150.32, 160.59, 171.48; MS (EI): $m / z(\%) 396\left(\mathrm{M}^{+}, 72\right)$. Anal. Calcd for $\mathrm{C}_{20} \mathrm{H}_{24} \mathrm{~N}_{6} \mathrm{OS}$ : C, 60.58; H, 6.10; N, 21.20. Found: C, 60.77; H, 6.32; N, 21.43.

4-(4-Methoxyphenyl)-2-(morpholinomethyl)-5-(pyridin-4yl)-2H-1,2,4-triazole-3(4H)-thione (6f) Yield: 75\%; mp: 182-184 ${ }^{\circ} \mathrm{C}$; IR $\left(\mathrm{KBr}, \mathrm{cm}^{-1}\right): 1610(\mathrm{C}=\mathrm{N}), 1255 \quad(\mathrm{C}=\mathrm{S})$; ${ }^{1} \mathrm{H}-\mathrm{NMR}$ (DMSO-d $)_{6} \delta: 2.93$ (s, 4H, 2 $\mathrm{CH}_{2}$ morpholine), 3.74 (s, 4H, 2 $\mathrm{CH}_{2}$ morpholine), $3.89\left(\mathrm{~s}, 3 \mathrm{H}, \mathrm{OCH}_{3}\right), 5.28(\mathrm{~s}, 2 \mathrm{H}$, $\mathrm{CH}_{2}$ ), 7.04-7.22 (m, 4H, Ar-H), 7.24 (d, 2H, $J=6 \mathrm{~Hz}$, pyridine $\mathrm{H}-3, \mathrm{H}-5), 8.60$ (d, 2H, J=6 Hz, pyridine $\mathrm{H}-2, \mathrm{H}-6) ;{ }^{13} \mathrm{C}-\mathrm{NMR}$ $\left(\mathrm{DMSO}-d_{6}\right) \delta: 50.84,55.59,66.91,77.35,115.28,121.57$, $127.09,129.29,132.91,147.06,150.40,160.67,171.68$; MS (EI): $m / z(\%) 383\left(\mathrm{M}^{+}\right.$, 84). Anal. Calcd for $\mathrm{C}_{19} \mathrm{H}_{21} \mathrm{~N}_{5} \mathrm{O}_{2} \mathrm{~S}: \mathrm{C}, 59.51$; H, 5.52; N, 18.26. Found: C, 59.78; H, 5.36; N, 18.42.

2-((Diethylamino)methyl)- 4 -(4-methoxy phenyl)-5 (pyridin-4-yl)-2H-1,2,4-triazol-3(4H)-thione (6g) Yield: 70\%; mp: $179-181^{\circ} \mathrm{C}$; IR $\left(\mathrm{KBr}, \mathrm{cm}^{-1}\right) ; 1608(\mathrm{C}=\mathrm{N}), 1261(\mathrm{C}=\mathrm{S})$; ${ }^{1} \mathrm{H}-\mathrm{NMR}\left(\mathrm{DMSO}-d_{6}\right) \delta$ : $1.04\left(\mathrm{t}, 6 \mathrm{H}, J=7.2 \mathrm{~Hz}, 2 \mathrm{CH}_{2}-\mathrm{CH}_{3}\right)$, 2.85 (q, 4H, J=7.2 Hz, $\left.2 \mathrm{CH}_{2}-\mathrm{CH}_{3}\right), 3.81\left(\mathrm{~s}, 3 \mathrm{H}, \mathrm{OCH}_{3}\right), 5.57$ (s, 2H, $\left.\mathrm{CH}_{2}\right), 7.06-7.14(\mathrm{~m}, 4 \mathrm{H}, \mathrm{Ar}-\mathrm{H}), 7.29$ (d, 2H, J=6 Hz, pyridine $\mathrm{H}-3, \mathrm{H}-5), 8.06$ (d, $2 \mathrm{H}, J=6 \mathrm{~Hz}$, pyridine $\mathrm{H}-2, \mathrm{H}-6)$; ${ }^{13} \mathrm{C}-\mathrm{NMR}$ (DMSO- $\left.d_{6}\right) \delta$ : 13.21, 45.43, 60.34, 76.72, 119.90, 126.41, 131.61, 134.05, 137.73, 152.05, 155.04, 165.28, 174.66; MS (EI): $m / z(\%) 369\left(\mathrm{M}^{+}, 64\right)$. Anal. Calcd for $\mathrm{C}_{19} \mathrm{H}_{23} \mathrm{~N}_{5} \mathrm{OS}$ : C, 61.76; H, 6.27; N, 18.95. Found: C, 61.98; H, 6.13; N, 19.01.

2-((4-Benzylpiperidin-1-yl)methyl)-4-(4-methoxyphenyl)5-(pyridin-4-yl)-2H-1,2,4-triazole-3(4H)-thione (6h) Yield: $78 \%$; mp: $161-163^{\circ} \mathrm{C}$; IR $\left(\mathrm{KBr}, \mathrm{cm}^{-1}\right): 1600(\mathrm{C}=\mathrm{N}), 1257$ $(\mathrm{C}=\mathrm{S}) ;{ }^{1} \mathrm{H}-\mathrm{NMR}\left(\mathrm{DMSO}-d_{6}\right) \delta: 1.24-1.71\left(\mathrm{~m}, 5 \mathrm{H}, 2 \mathrm{CH}_{2}+\mathrm{CH}\right.$ piperidine), 2.49 (s, $4 \mathrm{H}, 2 \mathrm{CH}_{2}$ piperidine), $3.23\left(\mathrm{~s}, 2 \mathrm{H}, \mathrm{CH}_{2}-\right.$ $\left.\mathrm{C}_{6} \mathrm{H}_{5}\right), 3.89\left(\mathrm{~s}, 3 \mathrm{H}, \mathrm{OCH}_{3}\right), 5.27\left(\mathrm{~s}, 2 \mathrm{H}, \mathrm{CH}_{2}\right), 7.02-7.25(\mathrm{~m}$, 9H, Ar-H), 7.28 (d, 2H, J=6 Hz, pyridine H-3, H-5), 8.58 (d, $2 \mathrm{H}, J=6 \mathrm{~Hz}$, pyridine $\mathrm{H}-2, \mathrm{H}-6) ;{ }^{13} \mathrm{C}-\mathrm{NMR}$ (DMSO- $\left.d_{6}\right) \delta$ : $32.15,37.44,43.15,51.32,55.58,70.37,115.22,121.56,125.82$, $127.20,128.16,129.08,129.34,133.06,140.52$, 146.78, 150.32, 160.60, 171.45; MS (EI): $m / z(\%) 471\left(\mathrm{M}^{+}, 29\right)$. Anal. Calcd for $\mathrm{C}_{27} \mathrm{H}_{29} \mathrm{~N}_{5} \mathrm{OS}$ : C, 68.76; H, 6.20; N, 14.85. Found: C, 68.54; H, $6.32 ; \mathrm{N}, 14.63$

4-(4-Chlorophenyl)-2-((4-methylpiperazin-1-yl)methyl)5-(pyridin-4-yl)-2H-1,2,4-triazole-3(4H)-thione (6i) Yield: 78\%; mp: $159-161^{\circ} \mathrm{C}$; IR $\left(\mathrm{KBr}, \mathrm{cm}^{-1}\right)$ : $1606(\mathrm{C}=\mathrm{N}), 1269$ $(\mathrm{C}=\mathrm{S}) ;{ }^{1} \mathrm{H}-\mathrm{NMR}\left(\mathrm{DMSO}-d_{6}\right) \delta: 1.27\left(\mathrm{~s}, 3 \mathrm{H}, \mathrm{CH}_{3}\right), 2.48(\mathrm{~s}, 4 \mathrm{H}$, $2 \mathrm{CH}_{2}$ of piperazine), $3.12\left(\mathrm{~s}, 4 \mathrm{H}, 2 \mathrm{CH}_{2}\right.$ of piperazine), 5.76 (s, 2H, $\left.\mathrm{CH}_{2}\right), 7.23-7.28(\mathrm{~m}, 4 \mathrm{H}, \mathrm{Ar}-\mathrm{H}), 7.52(\mathrm{~d}, 2 \mathrm{H}, J=6 \mathrm{~Hz}$, pyridine $\mathrm{H}-3, \mathrm{H}-5), 8.63(\mathrm{~d}, 2 \mathrm{H}, J=6 \mathrm{~Hz}$, pyridine $\mathrm{H}-2, \mathrm{H}-6)$; ${ }^{13} \mathrm{C}-\mathrm{NMR}$ (DMSO- $d_{6}$ ) $\delta$ : 46.01, 49.68, 54.55, 71.95, 121.58, 129.47, 130.12, 132.55, 132.75, 136.09, 146.68, 150.26, 169.51; MS (EI): $m / z(\%) 400\left(\mathrm{M}^{+}, 52\right)$. Anal. Calcd for $\mathrm{C}_{19} \mathrm{H}_{21} \mathrm{ClN}_{6} \mathrm{~S}$ : C, 56.92; H, 5.28; N, 20.96. Found: C, 56.67; H, 5.32; N, 21.03.

4-(4-Chlorophenyl)-2-(morpholinomethyl)-5-(pyridin-4yl)-2H-1,2,4-triazole-3(4H)-thione (6j) Yield: 75\%; mp: 139-141 ${ }^{\circ} \mathrm{C}$; IR $\left(\mathrm{KBr}, \mathrm{cm}^{-1}\right): 1597(\mathrm{C}=\mathrm{N}), 1278(\mathrm{C}=\mathrm{S})$; ${ }^{1} \mathrm{H}-\mathrm{NMR}$ (DMSO- $d_{6}$ ) $\delta: 2.90$ (s, 4H, 2 $\mathrm{CH}_{2}$ morpholine), 3.67 (s, 4H, 2 $\mathrm{CH}_{2}$ morpholine), $5.25\left(\mathrm{~s}, 2 \mathrm{H}, \mathrm{CH}_{2}\right), 7.20-7.28(\mathrm{~m}$, $4 \mathrm{H}, \mathrm{Ar}-\mathrm{H}), 7.50$ (d, 2H, $J=6 \mathrm{~Hz}$, pyridine H-3, H-5), 8.60 (d, $2 \mathrm{H}, J=6 \mathrm{~Hz}$, pyridine $\mathrm{H}-2, \mathrm{H}-6) ;{ }^{13} \mathrm{C}-\mathrm{NMR}$ (DMSO- $d_{6}$ ) $\delta$ : 50.81, 66.87, 70.26, 121.58, 129.51, 130.37, 132.57, 133.02, 136.47, 146.70, 150.52, 171.33; MS (EI): $m / z(\%) 387\left(\mathrm{M}^{+}, 54\right)$. Anal. Calcd for $\mathrm{C}_{18} \mathrm{H}_{18} \mathrm{ClN}_{5} \mathrm{OS}$ : C, 55.74; H, 4.68; N, 18.06. Found: C, 55.98; H, 4.36; N, 18.32. 
4-(4-Chlorophenyl)-2-((diethylamino)methyl)-5-(pyridin-4yl)-2H-1,2,4-triazole-3(4H)-thione (6k) Yield: 70\%; mp: $178-180^{\circ} \mathrm{C}$; IR $\left(\mathrm{KBr}, \mathrm{cm}^{-1}\right) ; 1606(\mathrm{C}=\mathrm{N}), 1269 \quad(\mathrm{C}=\mathrm{S})$; ${ }^{1} \mathrm{H}-\mathrm{NMR}\left(\mathrm{DMSO}-d_{6}\right) \delta$ : $1.24\left(\mathrm{t}, 6 \mathrm{H}, J=7.2 \mathrm{~Hz}, 2 \mathrm{CH}_{2}-\mathrm{CH}_{3}\right)$, 2.92 (q, 4H, J=7.2 Hz, $\left.2 \mathrm{CH}_{2}-\mathrm{CH}_{3}\right), 5.77$ (s, 2H, $\left.\overline{\mathrm{CH}}_{2}\right)$, $7.22-7.29(\mathrm{~m}, 4 \mathrm{H}, \mathrm{Ar}-\mathrm{H}), 7.53(\mathrm{~d}, 2 \mathrm{H}, J=6 \mathrm{~Hz}$, pyridine $\mathrm{H}-3$, $\mathrm{H}-5), 8.64$ (d, 2H, $J=6 \mathrm{~Hz}$, pyridine $\mathrm{H}-2, \mathrm{H}-6)$; MS (EI): $\mathrm{m} / \mathrm{z}$ (\%) $373\left(\mathrm{M}^{+}, 29\right)$. Anal. Calcd for $\mathrm{C}_{18} \mathrm{H}_{20} \mathrm{ClN}_{5} \mathrm{~S}$ : C, 57.82; H, 5.39; N, 18.73. Found: C, 57.65; H, 5.08; N, 18.91 .

2-((4-Benzylpiperidin-1-yl)methyl)-4-(4-chlorophenyl)-5(pyridin-4-yl)-2H-1,2,4-triazole-3(4H)-thione (6l) Yield: 78\%; mp: $166-168^{\circ} \mathrm{C}$; IR $\left(\mathrm{KBr}, \mathrm{cm}^{-1}\right)$ : $1598(\mathrm{C}=\mathrm{N}), 1267(\mathrm{C}=\mathrm{S})$; ${ }^{1} \mathrm{H}-\mathrm{NMR}\left(\mathrm{DMSO}-d_{6}\right) \delta: 1.46-1.71\left(\mathrm{~m}, 5 \mathrm{H}, 2 \mathrm{CH}_{2}+\mathrm{CH}\right.$ piperidine), 2.50 (s, $4 \mathrm{H}, 2 \mathrm{CH}_{2}$ piperidine), $3.23\left(\mathrm{~s}, 2 \mathrm{H}, \mathrm{CH}_{2}-\right.$ $\left.\mathrm{C}_{6} \mathrm{H}_{5}\right), 5.27\left(\mathrm{~s}, 2 \mathrm{H}, \mathrm{CH}_{2}\right), 7.12-7.29(\mathrm{~m}, 9 \mathrm{H}, \mathrm{Ar}-\mathrm{H}), 7 . \overline{51}(\mathrm{~d}$, $2 \mathrm{H}, J=6 \mathrm{~Hz}$, pyridine $\mathrm{H}-3, \mathrm{H}-5), 8.60$ (d, $2 \mathrm{H}, J=6 \mathrm{~Hz}$, pyridine $\mathrm{H}-2, \mathrm{H}-6) ;{ }^{13} \mathrm{C}-\mathrm{NMR}$ (DMSO-d $) \delta: 32.14,37.44,43.13,51.33$, $77.06,121.56,125.85,128.17,129.08,129.56,132.72,133.13$, $136.40,140.49,146.44,150.48,150.57,171.12$; MS (EI): $\mathrm{m} / \mathrm{z}$ (\%) $476\left(\mathrm{M}^{+}\right.$, 65). Anal. Calcd for $\mathrm{C}_{26} \mathrm{H}_{26} \mathrm{ClN}_{5} \mathrm{~S}$ : C, 65.60; H, 5.51; N, 14.71. Found: C, 65.42; H, 5.39; N, 14.64.

4-(4-Bromophenyl)-2-((4-methylpiperazin-1-yl)methyl)-5(pyridin-4-yl)-2H-1,2,4-triazole-3(4H)-thione (6m) Yield: $80 \%$; mp: $170-172^{\circ} \mathrm{C}$; IR $\left(\mathrm{KBr}, \mathrm{cm}^{-1}\right): 1606(\mathrm{C}=\mathrm{N}), 1271$ $(\mathrm{C}=\mathrm{S}) ;{ }^{1} \mathrm{H}-\mathrm{NMR}\left(\mathrm{DMSO}-d_{6}\right) \delta: 2.19\left(\mathrm{~s}, 3 \mathrm{H}, \mathrm{CH}_{3}\right), 2.43(\mathrm{~s}$, $4 \mathrm{H}, 2 \mathrm{CH}_{2}$ piperazine), $2.74\left(\mathrm{~s}, 4 \mathrm{H}, 2 \mathrm{CH}_{2}\right.$ piperazine), 5.77 (s, 2H, $\mathrm{CH}_{2}$ ), 7.20-7.28 (m, 4H, Ar-H), 7.69 (d, 2H, J=6 Hz, pyridine $\mathrm{H}-3, \mathrm{H}-5), 8.64$ (d, $2 \mathrm{H}, J=6 \mathrm{~Hz}$, pyridine $\mathrm{H}-2, \mathrm{H}-6)$; ${ }^{13} \mathrm{C}-\mathrm{NMR}\left(\mathrm{DMSO}-d_{6}\right.$ ) $\delta: 45.63,49.82,54.63,72.22,121.61$, 124.44, 129.70, 129.76, 132.56, 133.22, 146.88, 150.37, 169.52; MS (EI): $m / z(\%) 445\left(\mathrm{M}^{+}, 43\right)$. Anal. Calcd for $\mathrm{C}_{19} \mathrm{H}_{21} \mathrm{BrN}_{6} \mathrm{~S}$ : C, 51.24; H, 4.75; N, 18.87. Found: C, 51.44; H, 4.61; N, 18.99.

4-(4-Bromophenyl)-2-(morpholinomethyl)-5-(pyridin-4yl)-2H-1,2,4-triazole-3(4H)-thione (6n) Yield: 82\%; mp: $138-140^{\circ} \mathrm{C}$; IR $\left(\mathrm{KBr}, \mathrm{cm}^{-1}\right): 1598(\mathrm{C}=\mathrm{N}), 1276 \quad(\mathrm{C}=\mathrm{S})$; ${ }^{1} \mathrm{H}-\mathrm{NMR}$ (DMSO- $d_{6}$ ) $\delta: 2.91$ (s, 4H, 2 $\mathrm{CH}_{2}$ morpholine), 3.73 (s, 4H, 2 $\mathrm{CH}_{2}$ morpholine), $5.27\left(\mathrm{~s}, 2 \mathrm{H}, \mathrm{CH}_{2}\right), 7.20-7.23(\mathrm{~m}$, 4H, Ar-H), 7.68 (d, 2H, J=6 Hz, pyridine H-3, H-5), 8.63 (d, $2 \mathrm{H}, J=6 \mathrm{~Hz}$, pyridine $\mathrm{H}-2, \mathrm{H}-6) ;{ }^{13} \mathrm{C}-\mathrm{NMR}$ (DMSO- $\left.d_{6}\right) \delta$ : 50.81, 66.86, 70.25, 121.60, 124.59, 129.77, 132.57, 133.35, 133.54, 146.64, 150.51, 171.25; MS (EI): $m / z(\%) 432\left(\mathrm{M}^{+}, 53\right)$. Anal. Calcd for $\mathrm{C}_{18} \mathrm{H}_{18} \mathrm{BrN}_{5} \mathrm{OS}$ : C, 50.01; H, 4.20; N, 16.20. Found: C, 50.29; H, 4.40; N, 16.42 .

2-((Diethylamino)methyl)-4-(4-bromophenyl)-5-(pyridin-4yl)-2H-1,2,4-triazole-3(4H)-thione (6o) Yield: 75\%; mp: $178-180^{\circ} \mathrm{C}$; IR $\left(\mathrm{KBr}, \mathrm{cm}^{-1}\right): 1606(\mathrm{C}=\mathrm{N}), 1271(\mathrm{C}=\mathrm{S})$; ${ }^{1} \mathrm{H}-\mathrm{NMR}\left(\mathrm{DMSO}-d_{6}\right) \delta$ : $1.11\left(\mathrm{t}, 6 \mathrm{H}, J=7.2 \mathrm{~Hz}, 2 \mathrm{CH}_{2}-\mathrm{CH}_{3}\right)$, 3.33 (q, 4H, J=7.2 Hz, $\left.2 \mathrm{CH}_{2}-\mathrm{CH}_{3}\right), 5.57$ (s, $\left.2 \mathrm{H}, \overline{\mathrm{CH}}_{2}\right)$, 7.14-7.44 (m, 4H, Ar-H), $7.75(\mathrm{~d}, 2 \mathrm{H}, J=6 \mathrm{~Hz}$, pyridine H-3, $\mathrm{H}-5), 8.61$ (d, 2H, J=6 Hz, pyridine $\mathrm{H}-2, \mathrm{H}-6)$; ${ }^{13} \mathrm{C}-\mathrm{NMR}$ $\left(\mathrm{DMSO}-d_{6}\right) \delta$ : 13.02, 45.82, 71.93, 121.57, 124.24, 129.72, 132.50, 133.10, 133.27, 146.79, 150.28, 169.41; MS (EI): $\mathrm{m} / \mathrm{z}$ (\%) $417\left(\mathrm{M}^{+}\right.$, 90). Anal. Calcd for $\mathrm{C}_{18} \mathrm{H}_{20} \mathrm{BrN}_{5} \mathrm{~S}$ : C, 51.68; H, 4.82; N, 16.74. Found: C, 51.93; H, 4.61; N, 16.43.

2-((4-Benzylpiperidin-1-yl)methyl)-4-(4-bromophenyl)-5(pyridin-4-yl)-2H-1,2,4-triazole-3(4H)-thione (6p) Yield: 78\%; mp: $158-160^{\circ} \mathrm{C}$; IR $\left(\mathrm{KBr}, \mathrm{cm}^{-1}\right): 1598(\mathrm{C}=\mathrm{N}), 1267(\mathrm{C}=\mathrm{S})$; ${ }^{1} \mathrm{H}-\mathrm{NMR}\left(\mathrm{DMSO}-d_{6}\right) \delta: 1.26-1.76\left(\mathrm{~m}, 5 \mathrm{H}, 2 \mathrm{CH}_{2}+\mathrm{CH}\right.$ piperidine), 2.47 (s, 4H, $2 \mathrm{CH}_{2}$ piperidine), $3.22\left(\mathrm{~s}, 2 \mathrm{H}, \mathrm{CH}_{2}-\right.$ $\left.\mathrm{C}_{6} \mathrm{H}_{5}\right), 5.26\left(\mathrm{~s}, 2 \mathrm{H}, \mathrm{CH}_{2}\right), 7.12-7.29(\mathrm{~m}, 9 \mathrm{H}, \mathrm{Ar}-\mathrm{H}), 7 . \overline{66}(\mathrm{~d}$, $2 \mathrm{H}, J=6 \mathrm{~Hz}$, pyridine $\mathrm{H}-3, \mathrm{H}-5), 8.61(\mathrm{~d}, 2 \mathrm{H}, J=6 \mathrm{~Hz}$, pyridine
H-2, H-6); ${ }^{13} \mathrm{C}-\mathrm{NMR}$ (DMSO- $\left.d_{6}\right) \delta: 32.14,37.44,43.13,51.33$, $77.05,121.57,124.53,125.85,128.17,129.58,132.7,133.31$, 133.44, 133.65, 140.49, 146.39, 150.49, 150.58, 171.05; MS (EI): $m / z(\%) 520\left(\mathrm{M}^{+}\right.$, 47). Anal. Calcd for $\mathrm{C}_{26} \mathrm{H}_{26} \mathrm{BrN}_{5} \mathrm{~S}$ : C, 60.00; H, 5.03; N, 13.46. Found: C, 60.27; H, 5.32; N, 13.43.

In Vitro Cytotoxic Assay Chemicals Fetal bovine serum (FBS) and L-glutamine, were purchased from Gibco Invitrogen Co. (Scotland, U.K.). RPMI-1640 medium was purchased from Cambrex (NJ, U.S.A.). Dimethyl sulfoxide (DMSO), CHS 828, penicillin, streptomycin and sulforhodamine B (SRB) were purchased from Sigma Chemical Co. (Saint Louis, MO, U.S.A.).

Cell Cultures Cell cultures were obtained from the European Collection of cell Cultures (ECACC, Salisbury, U.K.) and human gastric cancer (NUGC), human colon cancer (DLD1), human liver cancer (HA22T and HEPG2), human breast cancer (MCF), nasopharyngeal carcinoma (HONE1) and normal fibroblast cells (WI38) were kindly provided by the National Cancer Institute (NCI, Cairo, Egypt). They were grown as monolayer and routinely maintained in RPMI-1640 medium supplemented with $5 \%$ heat inactivated FBS, $2 \mathrm{~mm}$ glutamine and antibiotics (penicillin $100 \mathrm{U} / \mathrm{mL}$, streptomycin $100 \mathrm{mg} / \mathrm{mL}$ ), at $37^{\circ} \mathrm{C}$ in a humidified atmosphere containing $5 \% \mathrm{CO}_{2}$. Exponentially growing cells were obtained by plating $1.5 \times 10^{5}$ cells $/ \mathrm{mL}$ for the six human cancer cell lines followed by $24 \mathrm{~h}$ of incubation. The effect of the vehicle solvent (DMSO) on the growth of these cell lines was evaluated in all the experiments by exposing untreated control cells to the maximum concentration $(0.5 \%)$ of DMSO used in each assay.

Conflict of Interest The authors declare no conflict of interest.

\section{References}

1) Lee Y. S., Park S. M., Kim H. M., Park S. K., Lee K., Lee C. W., Kim B. H., Bioorg. Med. Chem. Lett., 19, 4688-4691 (2009).

2) Harrison M. R., Holen K. D., Liu G., Clin. Adv. Hematol. Oncol., 7, 54-64 (2009)

3) Kumar D., Patel G., Chavers A. K., Chang K. H., Shah K., Eur. J. Med. Chem., 46, 3085-3092 (2011).

4) Patel N. B., Purohit A. C., Rajani D. P., Moo-Puc R., Rivera G., Eur. J. Med. Chem., 62, 677-687 (2013).

5) Rapolu S., Alla M., Bommena V. R., Murthy R., Jain N., Bommareddy V. R., Bommineni M. R., Eur. J. Med. Chem., 66, 91-100 (2013).

6) Gadegoni H., Manda S., Chin. Chem. Lett., 24, 127-130 (2013).

7) Rajak H., Agarawal A., Parmar P., Thakur B. S., Veerasamy R. Sharma P. C., Kharya M. D., Bioorg. Med. Chem. Lett., 21, 57355738 (2011).

8) Du Q. R., Li D. D., Pi Y. Z., Li J. R., Sun J., Fang F., Zhong W. Q., Gong H. B., Zhu H. L., Bioorg. Med. Chem., 21, 2286-2297 (2013).

9) Puthiyapurayil P., Poojary B., Chikkanna C., Buridipad S. K., Eur. J. Med. Chem., 53, 203-210 (2012).

10) Luo Z. H., He S. Y., Chen B. Q., Shi Y. P., Liu Y. M., Li C. W., Wang Q. S., Chem. Pharm. Bull., 60, 887-891 (2012).

11) Zhang F., Wang X. L., Shi J., Wang S. F., Yin Y., Yang Y. S., Zhang W. M., Zhu H. L., Bioorg. Med. Chem., 22, 468-477 (2014).

12) Farshori N. N., Banday M. R., Ahmad A., Khan A. U., Rauf A., Bioorg. Med. Chem. Lett., 20, 1933-1938 (2010).

13) Skrzypek A., Matysiak J., Niewiadomy A., Bajda M., Szymański P., Eur. J. Med. Chem., 62, 311-319 (2013).

14) Mishra G., Singh A. K., Jyoti K., Int. J. Chem. Tech. Res., 3, 13801393 (2011) 
15) Matysiak J., Opolski A., Bioorg. Med. Chem., 14, 4483-4489 (2006).

16) Matysiak J., Malinski Z., Bioorg. Khim., 33, 640-647 (2007).

17) Matysiak J., Nasulewicz A., Pelczynska M., Switalska M., Jaroszewicz I., Opolski A., Eur. J. Med. Chem., 41, 475-482 (2006).

18) Sahu J. K., Ganguly S., Kaushik A., Chin. J. Nat. Med., 11, 456465 (2013).

19) Koparir M., Orek C., Parlak A. E., Söylemez A., Koparir P., Karatepe M., Dastan S. D., Eur. J. Med. Chem., 63, 340-346 (2013).

20) Padmavathi V., Sudhakar Reddy G., Padmaja A., Kondaiah P., AliShazia, Eur. J. Med. Chem., 44, 2106-2112 (2009).

21) Shivarama Holla B., Narayana Poojary K., Sooryanarayana Rao B., Shivananda M. K., Eur. J. Med. Chem., 37, 511-517 (2002).

22) Clemons M., Coleman R. E., Verma S., Cancer Treat. Rev., 30, 324-332 (2004).

23) Sujith K. V., Rao J. N., Shetty P., Kalluraya B., Eur. J. Med. Chem., 44, 3697-3702 (2009).

24) Huczyński A., Rutkowski J., Borowicz I., Wietrzyk J., Maj E., Brzezinski B., Bioorg. Med. Chem. Lett., 23, 5053-5056 (2013).

25) Shivarama Holla B., Veerendra B., Shivananda M. K., Poojary B., Eur. J. Med. Chem., 38, 759-767 (2003).

26) Shaw A. Y., Chang C. Y., Hsu M. Y., Lu P. J., Yang C. N., Chen H. L., Lo C. W., Shiau C. W., Chern M. K., Eur. J. Med. Chem., 45, 2860-2867 (2010).

27) Hu G., Wang G., Duan N., Wen X., Cao T., Xie S., Huang W., Acta
Pharma. Sinica B, 2, 312-317 (2012).

28) Dimmock J. R., Chamankhah M., Allen T. M., Hallran S., Pharmazie, 50, 221-222 (1995).

29) Kamel M. M., Megally Abdo N. Y., Eur. J. Med. Chem., 86, 75-80 (2014).

30) Mishra R. K., Tewari R. K., J. Indian Chem. Soc., 68, 110-113 (1991).

31) Dhiman A. M., Wadodkar K. N., Indian J. Chem., 40B, 636-639 (2001).

32) Yar M. S., Akhter M. W., Acta Pol. Pharm. Drug Res., 66, 393-397 (2009).

33) Singh S., Sharma L. K., Saraswat A., Singh R. K. P., Monatsh. Chem., 143, 1427-1430 (2012).

34) Oruç E. E., Rollas S., Kandemirli F., Shvets N., Dimoglo A. S., J. Med. Chem., 47, 6760-6767 (2004).

35) Senthilvelan A., Thirumalai D., Ramakrishnan V. T., Tetrahedron, 60, 851-860 (2004)

36) Lee B., Larsen M., Kubiak T., Hyun L. B., Jane L. M., Maria K. T., U.S. Patent, 0171650A1 (2004).

37) Gülerman N. N., Doğan H. N., Rollas S., Johansson C., Çelik C., Farmaco, 56, 953-958 (2001).

38) Koparır M., Çetin A., Cansız A., Molecules, 10, 475-480 (2005).

39) Vig Hjarnaa P. J., Jonsson E., Latini S., Dhar S., Larsson R., Bramm E., Skov T., Binderup L., Cancer Res., 59, 5751-5757 (1999). 